\title{
A Fast GPU Algorithm for Complex Fenestration Systems Optimization
}

\author{
Ignacio Decia ${ }^{1}$, Eduardo Fernández ${ }^{1}$, Pablo Ezzatti ${ }^{1}$ \\ ${ }^{1}$ Instituto de Computación, Facultad de Ingeniería, Montevideo, Uruguay
}

\begin{abstract}
Choosing the right Complex Fenestration System (CFS) is a challenging problem when design goals and Climate-Based Daylight Modeling (CBDM) are taken into account. Current software tools, such as RADiance or EnergyPlus, only allow the evaluation of a given fenestration system, but lack support for automatically finding optimal designs. Furthermore, these tools are computationally expensive for daylight simulation.

In this work, we propose an optimization algorithm based on the Variable Neighborhood Search metaheuristic, and the daylight coefficient method for annual daylight simulation. Two strategies to compute the daylight coefficients are presented and implemented on a Graphics Processing Unit (GPU) on top of the Nvidia OptiX library.
\end{abstract}

Experimental results show our parallel GPU implementation provides fast and accurate results, which leads to significant reductions in simulation time (up to one order of magnitude) with respect to RADIANCE. The optimization test cases were successfully solved, and satisfactory solutions were found in reasonable time, allowing interactive design cycles.

\section{Introduction}

Daylighting design is an important issue in architectural design. Choosing a CFS that meets all designer goals is a difficult problem. In general, these design goals include a set of Lighting Intentions (LI) which are the illumination goals designers would like to achieve. Besides the imposed LI, restrictions can also include geometrical constraints over the CFS shape.

Traditional approaches to satisfy all LI require a complex process, often using the trial and error method until an acceptable solution is eventually found. Current software tools, such as RADIANCE (Larson and Shakespeare (1998)) or ENERgyPlus (EnergyPlus Development Team (2018)), only allow the evaluation of a given CFS, but lack support for automatically finding optimal designs. As a consequence, designers are restricted to choose from a reduced subset of all interesting CFS configurations. Some methods have been developed to solve the optimal CFS problem based on an optimization algorithm coupled to an external simulation tool for daylight computations (See, for example, Uribe et al. (2017), Rapone and Saro (2012)). Although these methods are capable of finding satisfactory solutions, they have long execution times (including precomputed steps if necessary).

On the other hand, in the last decade, GPUs have become an attractive platform for high performance computing due to their intrinsically parallel architecture and relatively low cost (Kirk and Wen-mei (2017)). Algorithms based on ray tracing techniques are highly parallel and well suited for this type of platforms. Recently there have been efforts focused on building interactive and real-time ray-tracing systems, such as the Nvidia OptiX engine framework (Parker et al. (2010)), designed to run on top Nvidia GPUs and other parallel architectures. Now, dedicated ray tracing acceleration hardware for fast ray traversal became available with the new Nvidia Turing GPU architecture (NVIDIA Corporation (2018)).

In this work, we formulate the problem of finding an optimal CFS as an optimization problem. A metaheuristic tool is presented for automated search of satisfactory solutions in the design space. The optimization variables are related to parameters defining the geometry of the CFS. The objective function may include energy or illuminance performance metrics. To guide the optimization process, we use the Variable Neighborhood Search (VNS) metaheuristic (Mladenović and Hansen (1997)). For fast annual daylight simulation, our method computes the Daylight Coefficients (DC) in parallel, using a Monte Carlo Ray Tracing algorithm implemented on top of the OptiX library.

\section{Background}

This section describes briefly the main OptiX features and the Monte Carlo Ray Tracing method used to 
compute the daylight coefficients.

\section{OptiX Library}

To create an OptiX application a set of programs (i.e., gpu kernels) must be implemented. These programs describe how rays are traced and the actions to be taken when a ray hits an object. The most relevant OptiX programs are explained below.

- Ray Generation program instances are created and executed in parallel in separate threads. Each instance creates and traces one or multiple primary rays that result in different light paths. A primary ray can also be associated with a data payload. If some data needs to be accumulated along the path, this payload can record the current state of the path and hold some accumulated value for the path vertices generated so far.

- Closest Hit programs are invoked when the closest intersection of a ray with the scene geometry is found. Typically, this program updates the data payload of the current ray and trace a new ray from the hit point to continue with the current light path.

- Any Hit programs are invoked for every ray-object intersection found along the traced ray.

- Miss programs are invoked when a ray does not intersect the scene geometry.

\section{Monte Carlo Ray Tracing}

Daylight coefficients describes the relationship between the outgoing radiance of sky patches and the incoming irradiance to a sensor (Tregenza and Waters (1983)). In order to obtain these coefficients, a method to compute the incoming irradiance must be adopted. In this work, we use a Monte Carlo Ray Tracing method based on the path integral formulation of the light transport (Veach (1998), Pharr et al. (2017)). The path integral formulation allow us to express the total incoming irradiance as a contribution of all light transport paths of different lengths. An advantage of this formulation is that Monte Carlo methods can be applied easily by sampling a set of random paths and averaging their individual irradiance contributions to obtain a final irradiance estimate. Only paths starting at the sensor and ending on an emissive surface have a non zero contribution. To generate paths, different sampling strategies can be used.

\section{Methodology}

\section{Overview}

In this section we present an algorithm to find satisfactory designs of indoor CFSs, taking into account both designer goals and CBDM as objective functions. In this work, only one type of CFS was implemented, but the method does not impose any restriction on a specific type. The CFSs studied correspond to opaque Venetian blinds composed of vertical or horizontal slats. The geometry is defined by the following set of parameters: blind thickness, tilt angle, inter-slat spacing, and slat thickness and width. See Figure 1.
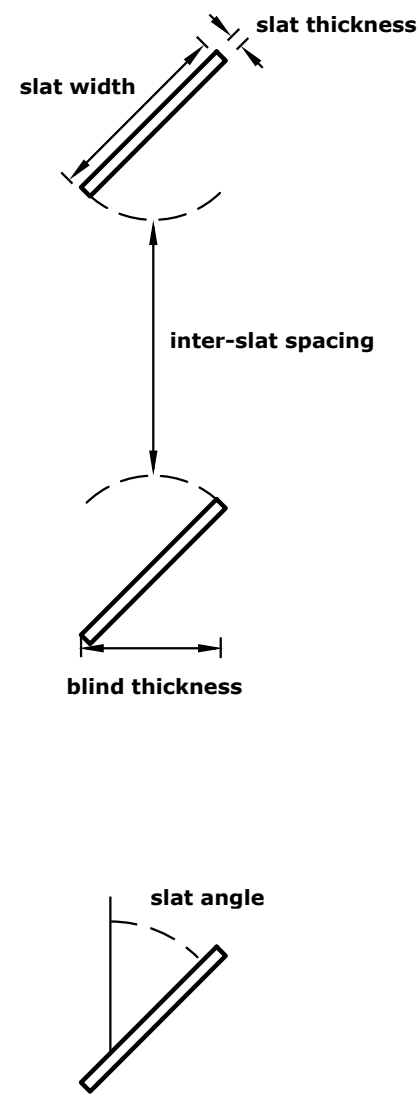

Figure 1: Parameters defining the geometry of a Venetian blind.

The CFS performance is evaluated doing annual daylight simulations based on the daylight coefficients computation of a set of indoor sensors. The algorithm starts from an initial parameter configuration, and progressively modifies these parameters until an acceptable solution is eventually found or some stopping criterion is satisfied. The VNS metaheuristic is adopted for efficient search space exploration.

\section{Problem Formulation}

The problem of finding an optimal CFS is formulated as the following optimization problem:

$$
\max _{x} \quad f(x)
$$

subject to:

$$
x_{i}^{m} \leq x_{i} \leq x_{i}^{M}, \quad i=1, \ldots, d .
$$

with $-\infty \leq x_{i}^{m} \leq x_{i}^{M} \leq \infty$. The decision variables $x=\left(x_{1}, \ldots, x_{d}\right)$ are a subset of the CFS parameters described previously. Some of them can 
also be fixed. The objective function $f(x)$ depends on annual daylight values and may include metrics related to building energy performance and visual comfort. The goal of the optimization problem is to find the optimal set of parameters in such a way that maximizes the objective function $f(x)$ subject to the box constraints.

\section{Daylight Coefficient Computation}

The most important part in the optimization process is the evaluation of CFSs, which depends directly on annual daylight simulations. Generally, daylight metrics take as input the simulated irradiance values of the sensors for a given set of sky conditions. Instead of running one independent simulation per sky condition, which would be prohibitive, the strategy is to compute the set of daylight coefficients for each sensor (Tregenza and Waters (1983)). After that, the total irradiance is obtained by multiplying these coefficients by the set of sky vectors containing the radiance value of each patch. This approach provides a fast method to do annual daylight simulations.

In this work, a set of $n$ sensors is defined by their positions and normal vectors. The sky is discretized into $m$ patches and let $s \in \mathbb{R}^{m}$ be a sky vector. A time series of $k$ sky conditions are grouped as column vectors into a sky matrix $S \in \mathbb{R}^{m \times k}$. The Daylight Coefficient Matrix, $D \in \mathbb{R}^{n \times m}$, is defined as the matrix such that each element $D_{i j}$ represents the fraction of flux leaving the sky patch $j$ that arrives at sensor $i$. Finally, the irradiance matrix, $E \in \mathbb{R}^{n \times k}$, whose elements $E_{i j}$ represent the total irradiance of sensor $i$ at sky condition $j$, is computed as follows:

$$
E=D \times S .
$$

We propose two methods to compute the daylight coefficients of a sensor. The first method, called One-Stage Method, is based on the Monte Carlo Ray Tracing method derived from the path integral formulation discussed before. In the second method, the flux transfer is divided into two stages. The first stage computes the outdoor flux transfer from sky to window, and the second stage computes the indoor flux transfer from window to sensor. We refer to this method as the Two-Stage Method. In this way, if only the indoor geometry changes, there is no need to recompute the outdoor flux.

\section{One-Stage Method}

In order to compute the daylight coefficients of a given sensor, we first define the emitted radiance of all sky patches as $1 \mathrm{~W} \cdot \mathrm{sr}^{-1} \cdot \mathrm{m}^{2}$. Subsequently, the daylight coefficients are obtained by computing in parallel the incoming irradiance from all sky patches. Applying the Monte Carlo Ray Tracing method, we trace $N$ paths in parallel GPU threads. When a sampled path hits the sky, the intersected sky patch number is recorded and its irradiance contribution is added to the corresponding coefficient. If a path did not hit the sky after a maximum number of bounces, the path is discarded, and its contribution is zero. Each sampled path has an OptiX data payload that records its current state and the sky patch number, in case the sky is intersected. A path is constructed incrementally, generating one vertex at a time. The first vertex starts always from a sensor. To determine the second vertex, the ray generation program samples a direction using a cosine density distribution over the hemisphere and traces a primary ray in that direction. At each vertex of the path, the closest hit program is responsible for updating the data payload and sampling a new direction using the cosine density distribution. The next vertex is found by tracing a ray from the current vertex in the sampled direction. If the traced ray misses all geometry, the miss program checks if the ray direction is pointing to the sky (positive $z$ axis). In that case, based on the ray direction, the corresponding sky patch number is stored in the data payload. Finally, after the path hits the sky, the ray generation program adds the irradiance contribution to the corresponding daylight coefficient.

\section{Two-Stage Method}

In the optimization process, each time some CFS parameters are modified, the local geometry changes and a recomputation of the daylight coefficients for all sensors is needed. Since we assume indoor CFSs, only the flux transfer from the window to the indoor space is affected. The outdoor flux transfer from sky to window remains unchanged. To avoid simulating the complete flux transfer again, the computation is broken in two stages. A matrix characterizes each stage of light transport. In the first stage, the flux relating the $m$ sky patches to the $k$ incident directions of the window is computed. This stage is executed only once, before the optimization phase starts. The output of this stage is the Outdoor Environment Matrix, $O \in \mathbb{R}^{k \times m}$, containing the flux coefficients related to the outdoor environment. In the second stage, the flux relating the $k$ window directions and the $n$ sensors is computed. This stage is executed each time a CFS needs to be evaluated. The output of this stage is the Indoor Environment Matrix, $I \in \mathbb{R}^{n \times k}$, containing the flux coefficients related to the indoor environment. Multiplying both matrices we obtain the daylight coefficients matrix $D \in \mathbb{R}^{n \times m}$,

$$
D=I \times O .
$$

\section{Outdoor Environment Matrix Computation}

Each element $O_{i j}$ represents the fraction of flux leaving the sky patch $j$ that arrives at the window 
from the incoming $i$ direction. We have chosen the B\&B Hemisphere division (Beckers and Beckers (2012)) to describe the incoming directions, since it divides the hemisphere into $k$ equal area and similar aspect ratio patches. Therefore, these patches have very comparable forms, i.e., close to the square. Each patch defines a solid angle around an incoming direction. The coefficients of a given B\&B patch correspond to the average radiance from the incoming directions defined by its solid angle. The algorithm computes the coefficients of all patches in parallel. For this purpose, based on the path integral formulation, we implemented a Monte Carlo Ray Tracing method in a similar way as we have already done for the irradiance computation. Applying this method, we trace $N$ paths in parallel GPU threads. When a sampled path hits the sky, the intersected sky patch number is recorded and the path contribution is added to the corresponding coefficient. The first vertex starts from the center of the window and the second vertex is determined by uniform sampling the $\mathrm{B} \& \mathrm{~B}$ hemisphere. The rest of the vertices are determined by sampling directions using the cosine density distribution. When a path with initial direction $i$ ends on the sky patch $j$, we add its contribution to the $O_{i j}$ entry.

\section{Indoor Environment Matrix Computation}

Each element $I_{i j}$ represents the fraction of flux leaving the window in the outgoing $j$ direction that arrives at sensor $i$. In order to obtain the coefficients of a given sensor, we compute the incoming irradiance from all window directions in parallel. Applying the Monte Carlo Ray Tracing method, we trace $N$ paths in parallel GPU threads. When a sampled path hits the window, the incoming direction is recorded and its contribution is added to the corresponding coefficient. All vertices of a path are generated sampling directions using the cosine distribution, except for the last vertex, which is generated by sampling the window surface explicitly, using an area density distribution defined over its surface. When a path starting from sensor $i$ arrives at the window from the $j$ direction, we add its contribution to the $I_{i j}$ entry.

\section{Variable Neighborhood Search}

We have chosen the VNS as the optimization algorithm, which is a widely used metaheuristic to solve a large variety of optimization problems (Mladenović and Hansen (1997)). This method is based on the successive exploration of a set of neighborhoods $N_{1}(x), N_{2}(x), \ldots, N_{k_{\max }}(x)$ to find the best solution. Each neighborhood $N_{i}(x)$ defines a set of candidate solutions around the solution $x$. The algorithm starts from an initial solution $x$, and then computes a local search on the first neighborhood $N_{1}(x)$. If a better solution is not found in the current neighborhood $N_{l}(x)$, the neighborhood structure is changed and the search continues in the next neighborhood $N_{l+1}(x)$; and so on. Otherwise, if a better solution $x^{\prime}$ is found on the current neighborhood $N_{l}(x)$, the current solution $x$ is replaced by $x^{\prime}$, and the local search is restarted in the first neighborhood $N_{1}\left(x^{\prime}\right)$. The algorithm usually ends when a maximum number of function evaluations is reached or when all the neighborhoods are explored, and a better solution could not be found. Typically, the neighborhoods used in VNS are nested: $N_{1}(x) \subset N_{2}(x) \subset \ldots \subset N_{k_{\max }}(x)$.

In our implementation, the decision variables of the solution $x$ are the geometry parameters defining the CFS. Each neighborhood $N_{k}$ takes into account two parameters: the number of decision variables that can be modified at the same time $(v)$, and a discretization $(r)$ of the normalized range value of the variable that is being modified. For instance, when $v=1$ and $r=0.1$, a single decision variable $x_{i}$ from $x$ is randomly selected, and then a new value of $x_{i}$ is chosen from the interval $x_{i} \pm 0.1\left(x_{i}^{M}-x_{i}^{m}\right)$, where $x_{i}^{M}$ and $x_{i}^{m}$ are the maximum and minimum possible values for $x_{i}$, respectively. In one step, a variable related to the slat angle is modified; in the next step, a variable related to slat width is modified; and so on.

\section{Experimental Evaluation}

This section describes the experimental study to test our method implementations.

\section{Hardware Environment}

All tests were executed on a PC with an Intel Core i5-7550 at $3.4 \mathrm{GHz}$., 8GB RAM, connected to an Nvidia Geforce 1060 GPU with 3GB RAM.

\section{Daylight Coefficient Test Case}

The goal of the experiment is to show the effectiveness of performing daylight computations on GPU by comparing the efficiency of RADIANCE with the One-Stage Method.

The test scene used, shown in Figure 2, is composed of an office with an indoor window of $2 \times 1.5 \times 0.25 \mathrm{~m}$ facing a lightwell. All materials are diffuse, with a reflectance of $80 \%$, except for the ground which is $20 \%$. The sky was discretized using the Tregenza 145-patch scheme.

The test case consisted in computing the daylight coefficients matrix corresponding to three indoor sensors. Their locations are shown in Figure 2. For each sensor we traced $N=10^{7}$ paths with a maximum of 30 bounces. The same configuration was used by RADIANCE and the One-Stage Method.

Let us denote by $D^{r a d}, D^{o p t} \in \mathbb{R}^{3 \times 145}$ the daylight coefficients matrices returned by RADIANCE and the One-Stage Method, respectively. The mean relative difference (MRD) between both matrices entries is $4.5 \%$. Figure 3 shows the plot of the coefficients 

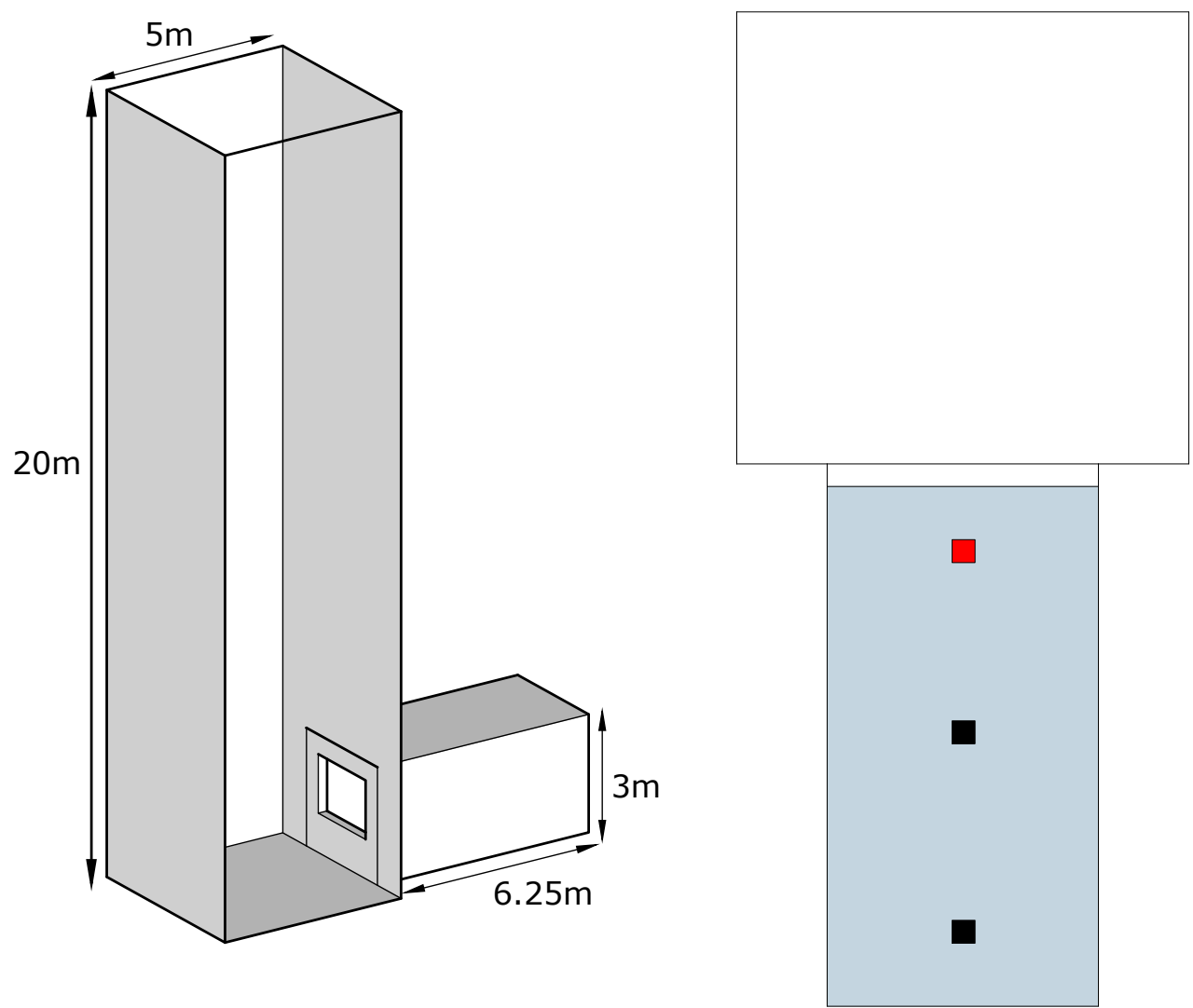

Figure 2: Left: Lightwell scene used for the DC computation test case. Right: Lightwell top view. The tiny squares are sensors located $0.7 \mathrm{~m}$ above the ground. The red square is sensor $I$.

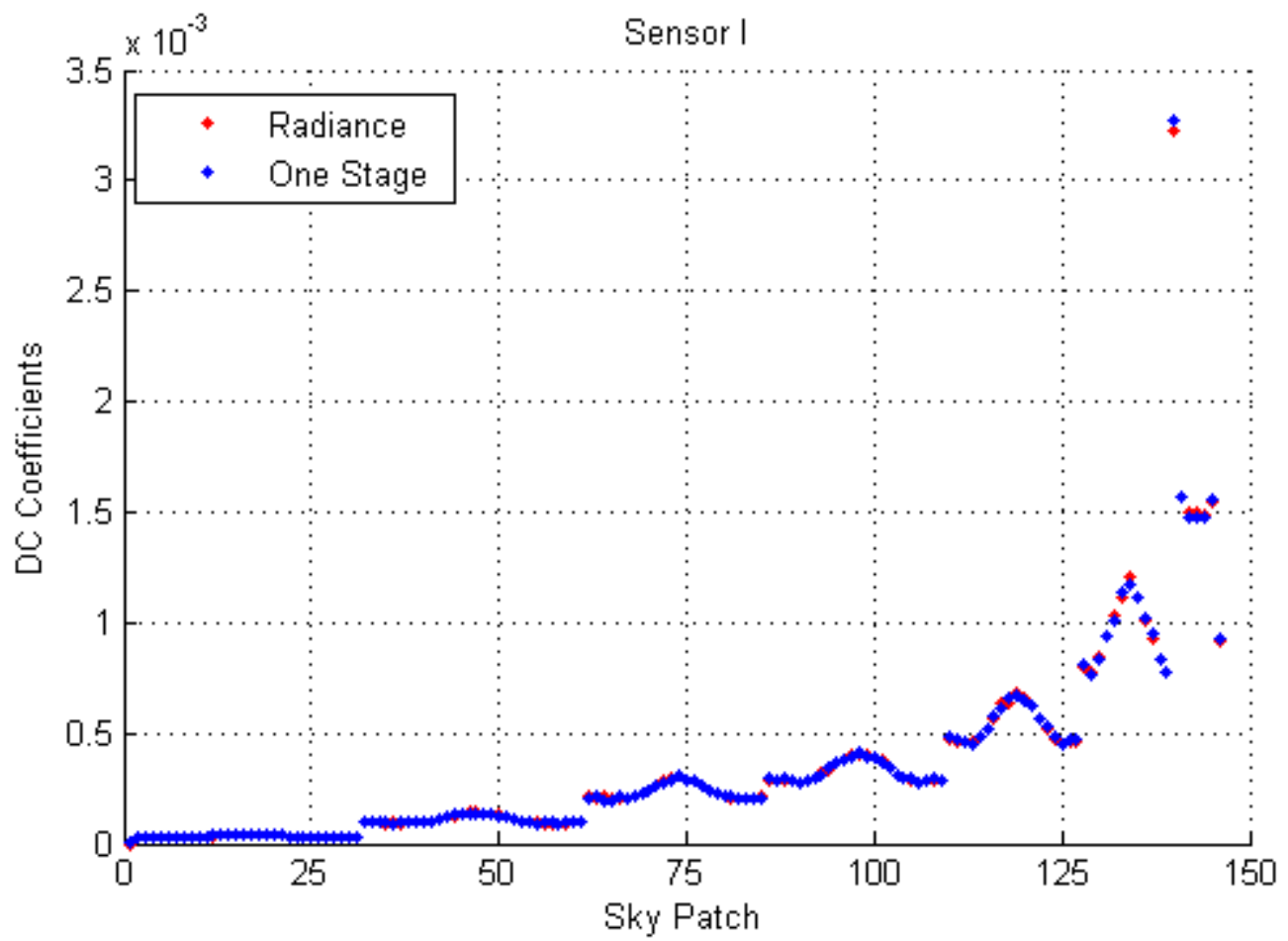

Figure 3: Comparison of DC of sensor I (red quare) between RADIANCE and the One-Stage method. Both methods seems to converge to the same values. 

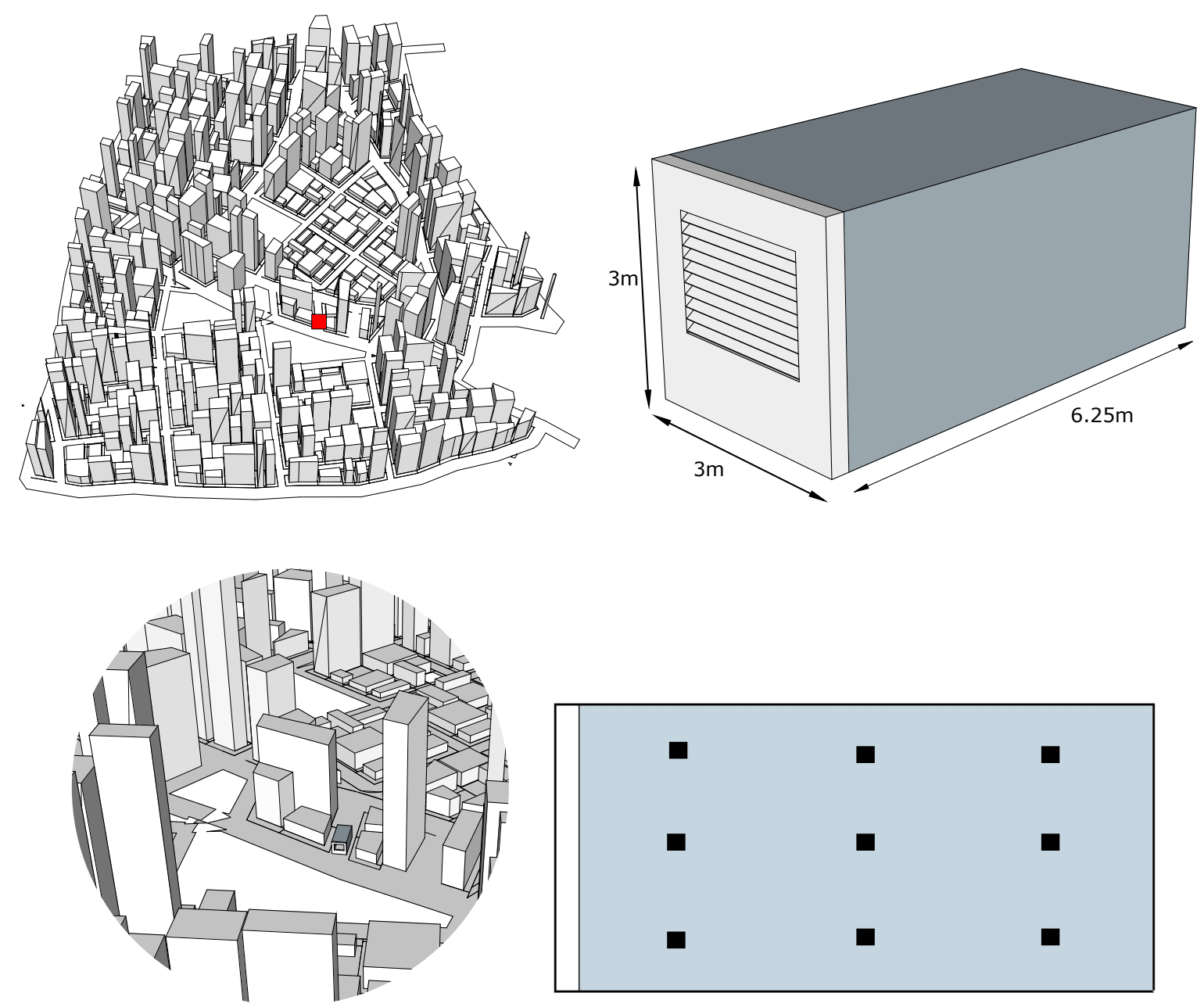

Figure 4: Top: City and office used for the optimization test case. The office is located at the red square shown in the city figure. Bottom: Magnified view of the city near the office and office top view. The tiny squares are sensors located $0.7 \mathrm{~m}$ above the ground.
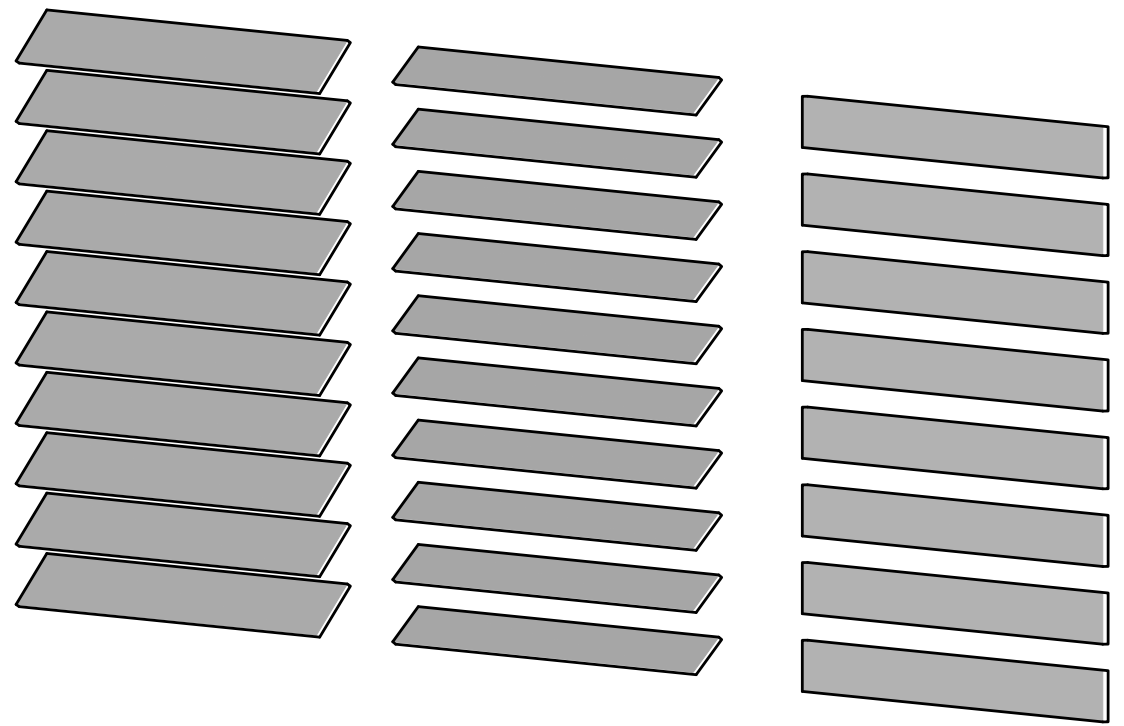

Figure 5: Three solutions found by the algorithm. Left blind: $U D I_{[100,3000]}=32 \%$, slat angle $\alpha=0.58 \mathrm{rad}$, slat width $w=0.15 \mathrm{~m}$, inter-slat spacing $s=0 \mathrm{~m}$ and slat thickness $t=0.01 \mathrm{~m}$. Middle blind: $U D I_{[100,3000]}=32 \%$, $\alpha=0.67 \mathrm{rad}, w=0.11 \mathrm{~m}, \mathrm{~s}=0.044 \mathrm{~m}$ and $t=0.01 \mathrm{~m}$. Right blind: $U D I_{[100,3000]}=37 \%, \alpha=0.00 \mathrm{rad}, w=0.13 \mathrm{~m}$, $s=0.07 \mathrm{~m}$ and $t=0.014 \mathrm{~m}$. In order to fit on the window, this blind has fewer slats. 
of sensor I. As we can see, both methods converge (almost) to the same values. Other tests also show that the MRD is further reduced by increasing the number of sampled paths.

Although these results show the convergence of the One-Stage Method, they reveal nothing about the relative efficiency between RADIANCE and our method. In order to compare both methods, we need a quality measure that takes into account both the variance and the computation time. For this purpose, the following metric described in Owen (2013) is adopted. Suppose we have two unbiased estimators $\widehat{\mu_{0}}, \widehat{\mu_{1}}$ of the same quantity $\mu$, with variances $\sigma_{0}^{2} / n$ and $\sigma_{1}^{2} / n$, respectively. Both methods take $n$ samples to produce a single $\mu$ estimate, and each sample is generated at an average $\operatorname{cost}$ of $c_{0}$ and $c_{1}$, respectively. The relative efficiency $Q$ between both methods is defined as:

$$
Q=\frac{c_{0} \sigma_{0}^{2}}{c_{1} \sigma_{1}^{2}}
$$

At the same level of accuracy, the first method takes $Q$ times as much work as the second. The efficiency value has two ratios: $\sigma_{0}^{2} / \sigma_{1}^{2}$ and $c_{0} / c_{1}$. The former is a mathematical property related to the accuracy of the estimates and the latter is related to the computational effort of the sampling process, which depends heavily on the algorithm implementations.

Continuing with our problem, let $D_{i j}^{o p t}, D_{i j}^{r a d}$ denote the daylight coefficient estimators corresponding to sensor $i$ and sky patch $j$. According to the above formula, their relative efficiency is given by $Q_{i j}$, for all $i, j$. We take the average relative efficiency $\widehat{Q}=\frac{1}{M} \sum Q_{i j}$, where $M$ is the total number of coefficients. We have calculated experimentally each term $Q_{i j}$ and obtained an average sampling cost ratio $c_{i j}^{r a d} / c_{i j}^{o p t}$ of 49.9 , and an average variance ratio $\left(\sigma_{i j}^{r a d}\right)^{2} /\left(\sigma_{i j}^{o p t}\right)^{2}$ of 0.24 . Therefore, if we fix the number of samples, our method is approximately fifty times faster than RADIANCE, but in order to achieve the same level of accuracy, our method needs approximately four times more samples. As a result, the average relative efficiency $\widehat{Q}$ between RADIANCE and our method is approximately 11.9.

\section{Optimization Algorithm Test Case}

The goal of the second experiment is to test the optimization algorithm and the Two-Stage method.

The test scene, shown in Figure 4, is composed of a city and the same office of the previous test case. Hourly sky and Sun conditions were derived from the direct normal and diffuse horizontal irradiation data, extracted from Test Reference Year Data (available at https://energyplus.net/weather). The geographical location is Montevideo-Uruguay $\left[34^{\circ} 49^{\prime} \mathrm{S}, 56^{\circ} 0^{\prime} \mathrm{W}\right]$. The room window faces to north. Both city and office materials are diffuse. The city has a reflectance of $20 \%$. The office has a reflectance of $80 \%$, except for its ground which is $20 \%$. We used the Tregenza 145-patch scheme and the B\&B Hemisphere (with 288 patches) to describe incoming directions to the window.

The precomputation of the outdoor matrix took 5 seconds tracing $N=2 \times 10^{7}$ paths. The maximum number of bounces was set to 30 . To compute the indoor matrix, for each sensor we traced $N=2 \times 10^{5}$ paths and set the maximum number of bounces to 5 . The first optimization problem consisted in finding the optimal slat angle $\alpha \in[0, \pi / 2]$ of an indoor Venetian blind in order to maximize the Useful Daylight Illuminance (UDI) metric considering nine indoor sensors. The $\mathrm{UDI}_{[a, b]}$ is defined as the percentage of hours of the year that the incoming illuminance to all sensors is between $a$ and $b$ (Nabil and Mardaljevic (2005)). For this problem we set $a=100 \mathrm{~lx}$ and $b=3000 \mathrm{~lx}$.

The optimization algorithm started from an initial solution with $\alpha=0.0 \mathrm{rad}$ and $\mathrm{UDI}_{[100,3000]}=0 \%$. The other blind parameters were fixed. The stopping criterion for the optimization algorithm was to reach 150 function evaluations. After 71 seconds the best solution found by the algorithm was $\alpha=0.58 \mathrm{rad}$ with $\mathrm{UDI}_{[100,3000]}=32 \%$.

To test the converge of the optimization algorithm we imposed the following related inverse problem: find the slat angle $\alpha \in[0, \pi / 2]$ that gives an $\mathrm{UDI}_{[100,3000]}=32 \%$. From the latter result we know that the optimal solution is given by $\alpha^{*}=0.58 \mathrm{rad}$. After 64 seconds the best solution found by the algorithm was $\alpha=0.57 \mathrm{rad}$ with $\mathrm{UDI}_{[100,3000]}=32 \%$, which has the same fitness value as the optimal solution. Continuing with our inverse problem, we added the slat width $w \in[0.1,0.15]$ as another optimization variable. After 69 seconds the algorithm now returned $\alpha=0.67 \mathrm{rad}$ and $w=0.11 \mathrm{~m}$ with $\mathrm{UDI}_{[100,3000]}=32 \%$.

Finally, the last problem consisted in maximizing the UDI taking into account four variables: slat angle $\alpha \in[0, \pi / 2]$, slat width $w \in[0.05,0.15]$, inter-slat spacing $s \in[0,0.1]$ and slat thickness $t \in[0.01,0.02]$. The stopping criterion was increased to 500 function evaluations. The optimization algorithm started from an initial solution with $\mathrm{UDI}_{[100,3000]}=0 \%$. After 373 seconds the algorithm returned $\alpha=0.00 \mathrm{rad}$, $w=0.13 \mathrm{~m}, s=0.07 \mathrm{~m}$ and $t=0.014 \mathrm{~m}$ with $\mathrm{UDI}_{[100,3000]}=37 \%$. Regarding to the first test case, a relative improvement in the UDI value of $15.6 \%$ is obtained.

In conclusion, for all the test cases performed the algorithm found a satisfactory solution in a few minutes, making possible interactive design cycles. These examples also showed that several parameter configurations can achieve a similar performance. Figure 5 shows the geometry of the solutions found. 


\section{Conclusions and Future Work}

The goal of this work is to optimize the geometry of an indoor CFS taking into account designer restrictions and CBDM metrics. We proposed an optimization algorithm and discussed its implementation in detail. The algorithm is based on the VNS metaheuristic and the daylight coefficient method for annual daylight simulations. Two different strategies to compute in parallel the daylight coefficients were presented. These strategies were implemented on GPU on top of the Nvidia OptiX library. The Two-Stage method proved to be useful for indoor geometry optimization problems since it avoided the complete computation of the flux transfer. The experimental results showed that our GPU implementations provided fast and accurate results, achieving a relative efficiency up to one order of magnitude with respect to RADIANCE. The optimization scenarios tested were successfully solved and satisfactory solutions were found in reasonable time.

As future work, we plan exhaustive evaluations, including comparisons with matrix-based methods (Three-Phase Method). A second line of work is to test other light path sampling strategies, such as bidirectional path tracing or light tracing. Stratified sampling can be used to get a more even distribution of the primary rays. Finally, population-based metaheuristics like evolutionary algorithms should be tested.

\section{Acknowledgment}

This work was partially funded by the FSE_1_2017_1_144731 project from Agencia Nacional de Investigación e Innovación (ANII, Uruguay). The authors gratefully thank to Antoine Bugeat for his support with RADIANCE.

\section{References}

Beckers, B. and P. Beckers (2012). A general rule for disk and hemisphere partition into equal-area cells. Computational Geometry 45(7), $275-283$.

EnergyPlus Version 9.0.1 (2018). Input Output Reference: The Encyclopedic Reference to EnergyPlus Input and Output.

Kirk, D. B. and W. H. Wen-mei (2017). Programming Massively Parallel Processors (Third ed.). Morgan Kaufmann.

Larson, G. W. and R. Shakespeare (1998). Rendering with Radiance: The Art and Science of Lighting Visualization. San Francisco, CA, USA: Morgan Kaufmann Publishers Inc.

Mladenović, N. and P. Hansen (1997, November). Variable neighborhood search. Comput. Oper. Res. 24(11), 1097-1100.
Nabil, A. and J. Mardaljevic (2005). Useful daylight illuminance: a new paradigm for assessing daylight in buildings. Lighting Research \& Technology 37(1), 41-57.

NVIDIA Corporation (2018). NVIDIA Turing GPU Architecture Whitepaper.

Owen, A. B. (2013). Monte Carlo theory, methods and examples.

Parker, S. G., J. Bigler, A. Dietrich, H. Friedrich, J. Hoberock, D. Luebke, D. McAllister, M. McGuire, K. Morley, A. Robison, and M. Stich (2010, August). Optix: A general purpose ray tracing engine. ACM Transactions on Graphics.

Pharr, M., W. Jakob, and G. Humphreys (2017). Physically Based Rendering (Third Edition). San Francisco, CA, USA: Morgan Kaufmann.

Rapone, G. and O. Saro (2012). Optimisation of curtain wall faades for office buildings by means of pso algorithm. Energy and Buildings 45, 189 196.

Tregenza, P. and I. Waters (1983). Daylight coefficients. Lighting Research \&5 Technology 15(2), 65-71.

Uribe, D., W. Bustamante, and S. Vera (2017). Seasonal optimization of a fixed exterior complex fenestration system considering visual comfort and energy performance criteria. Energy Procedia 132, 490 - 495. 11th Nordic Symposium on Building Physics, NSB2017, 11-14 June 2017, Trondheim, Norway.

Veach, E. (1998). Robust Monte Carlo Methods for Light Transport Simulation. Ph. D. thesis, Stanford, CA, USA. AAI9837162. 